\title{
Agregação de Cadeias de Acetatos de Celulose em LiCl/DMAc: Avaliação via Viscosimetria
}

\author{
Daniella L. Morgado, Virginia da C. A. Martins, Ana M. de G. Plepis, Elisabete Frollini \\ Instituto de Química de São Carlos, USP
}

\begin{abstract}
Resumo: Celulose de linter foi acetilada, visando obter acetatos de celulose com diferentes Graus de Substituição (GS) em meio homogêneo, usando cloreto de lítio/N,N-dimetilacetamida (LiCl/DMAc) como sistema de solvente, e anidrido acético como reagente acetilante. A agregação entre cadeias de celulose ou acetatos de celulose (GS 0,8, 1,5 e 2,0) em solução foi avaliada através de medidas viscosimétricas. Os resultados mostraram que a formação de agregados no sistema de solvente utilizado (LiCl/DMAc) é diferente para celulose e acetatos, e dependente da temperatura e do GS, no caso dos acetatos. Este trabalho corresponde à primeira etapa de um estudo em que se pretende preparar filmes de acetatos, assim como de acetatos reforçados com celulose, diretamente a partir de soluções destes em LiCl/DMAc. Os resultados apresentados permitem uma escolha melhor embasada do intervalo de concentração mais adequado para preparação de filmes, a partir de soluções de acetatos e celulose nesse sistema de solvente.
\end{abstract}

Palavras-chave: Celulose de linter, acetato de celulose, sistema de solvente LiCl/ DMAc, agregação.

\section{Aggregation of Chains of Cellulose Acetates in LiCl/DMAc: Evaluation Via Viscometry}

\begin{abstract}
Linters cellulose was acetylated to obtain cellulose acetates with different degrees of substitution (DS) in homogeneous medium, using lithium chloride/N,N-dimethylacetamide (LiCl/DMAc) as solvent system, and acetic anhydride as acetylating agent. The aggregation among chains of cellulose or cellulose acetates (DS 0.8, 1.5 and 2.0) in solution was studied through viscometric measurements. The results showed that aggregation in $\mathrm{LiCl} / \mathrm{DMAc}$ is different for cellulose and acetates, and depends on the temperature and DS in the case of acetates. This study corresponds to the first step of a project, which aims at to prepare cellulose acetates films, as well as acetates films reinforced with cellulose, by casting directly from these solutions in LiCl/DMAc. The results presented here allow for an optimized choice of concentration range for preparing films from LiCl/DMAc solutions of acetates and cellulose.
\end{abstract}

Keywords: Linters cellulose, cellulose acetate, solvent system LiCl/DMAc, aggregation.

\section{Introdução}

A celulose é um biopolímero de grande interesse devido à sua abundância na natureza e biodegradabilidade, sendo usada em diversas aplicações ${ }^{[1,2]}$. No entanto, devido à presença de ligações hidrogênio intra- e intermolecular entre suas cadeias, não é solúvel em solventes comuns. A substituição dos grupos hidroxilas presentes nas cadeias de celulose (Figura 1a) por grupos menos polares, visando solubilização pós derivatização, é bastante comum ${ }^{[3]}$. Embora estes recursos sejam usados com certa frequência, nem sempre é levado em conta que a dissolução de derivados de celulose também pode não levar a uma dispersão molecular, mas a um estado coloidal, consistindo de cadeias $\operatorname{agregadas}^{[4]}$.

A formação de agregados (processo irreversível) ou associados (processo reversível) de celulose e seus derivados em solução pode ser investigada através de medidas viscosimétricas ${ }^{[5]}$. No entanto, pode-se considerar que muitos aspectos referentes aos processos de agregação e/ou associação ainda precisam ser investigados. $\mathrm{Na}$ presente investigação, buscou-se contribuir para com o estudo da formação de agregados e/ou associados de celulose e acetatos de celulose em LiCl/DMAc, sendo este estudo realizado via viscosimetria. Na sequência, tem-se como meta a preparação de filmes de acetato de celulose e acetato de celulose reforçado por celulose, a partir deste sistema de solvente.

\section{Experimental}

A celulose utilizada foi a de linter de algodão, de baixa massa molar média (usada para fins têxteis), gentilmente cedida pela Indústria Fibra S.A. (Americana, São Paulo), tendo sido submetida a um pré-tratamento com solução alcalina, denominado mercerização, necessário para solubilizar a mesma em $\mathrm{LiCl} / \mathrm{DMAc}$, seguindo o procedimento descrito em trabalho anterior $^{[6]}$. Os acetatos de celulose foram sintetizados em $\mathrm{LiCl} / \mathrm{DMAc}$ e caracterizados seguindo o procedimento descrito previamente ${ }^{[7]}$, o mesmo ocorrendo com o Grau de Substituição (GS) dos acetatos de celulose, determinado por ${ }^{1} \mathrm{H} \mathrm{NMR}^{[7]}$.

Viscosimetria de soluções de celulose de linter e acetatos de celulose em LiCl/DMAc: Preparou-se inicialmente a solução de $\mathrm{LiCl} / \mathrm{DMAc}$ com concentração de 5,3\% em massa, à $80^{\circ} \mathrm{C}$, sob agitação durante o período de 1 hora. $\mathrm{O}$ linter mercerizado e os acetatos foram dissolvidos na concentração desejada (solução estoque), seguindo o procedimento descrito em trabalho anterior $^{[8]}$. Após 24 horas, a solução estoque foi então filtrada, sob pressão positiva, em membrana de vidro de $47 \mathrm{~mm}$ de diâmetro, não havendo retenção de material no filtro. Em seguida, soluções diluídas foram preparadas. Utilizou-se um reômetro TA AR 1000-N, ligado a um banho termostático Julabo, FS 185. As soluções foram analisadas utilizando-se a geometria Couette, sistema DG41 (dois cilindros concêntricos com espaço entre os raios internos de $3,95 \mathrm{~mm}$ ), no intervalo de velocidade de cisalhamento de $0,1-1000 \mathrm{~s}^{-1}$, à temperatura 25 e $50^{\circ} \mathrm{C},\left( \pm 1,0^{\circ} \mathrm{C}\right)$.

\section{Resultados e Discussão}

O GS pode ser calculado através da relação entre a área correspondente à ressonância dos prótons do anel de glicose

Autor para correspondência: Elisabete Frollini, Instituto de Química de São Carlos, Universidade de São Paulo, Caixa Postal 780, CEP: 13560-970,

São Carlos, SP, Brasil. E-mail: elisabete@iqsc.usp.br 
( $\delta \sim 2,90-5,10 \mathrm{ppm})$ e a área correspondente à ressonância dos prótons metílicos do grupo acetato $(\delta \sim 1,70-2,20 \mathrm{ppm})^{[9]}$. A análise dos acetatos de linter, previamente sintetizados no sistema de solvente $\mathrm{LiCl} / \mathrm{DMAc}$, usando os espectros de ${ }^{1} \mathrm{H}$ RMN (figuras não mostradas), levou a valores de GS de 0,8; 1,5 e 2,0.

Em relação ao efeito da velocidade de cisalhamento, observou-se que todas as soluções de celulose e de acetatos de celulose analisadas apresentaram comportamento newtoniano, ou seja, a viscosidade das soluções é independente da velocidade de cisalhamento (figuras não mostradas). Os valores da viscosidade intrínseca [ $\eta]$ e constante de Huggins $\left(\mathrm{k}_{\mathrm{H}}\right)$ foram obtidos a partir dos gráficos de $\frac{\eta_{\mathrm{sp}}}{\mathrm{c}}$ versus concentração (sendo $\eta_{\mathrm{sp}}$ a viscosidade específica, c a concentração, e $[\eta]$ e $\mathrm{k}_{\mathrm{H}}$ obtidos a partir dos coeficientes linear e angular da curva, respectivamente, figuras não mostradas) ${ }^{[10]}$.

A presença dos grupos hidroxila na estrutura da celulose (Figura 1b, I) pode levar a agregação entre as cadeias, até mesmo em sistemas supostamente diluídos, ou seja, a celulose pode não estar presente como cadeias individualmente solvatadas, mas como agregados solvatados ${ }^{[1]}$, dificultando a obtenção de soluções "verdadeiras". Mesmo se encontrando correlações lineares entre viscosidade reduzida e concentração (figuras não mostradas), os valores da constante de Huggins $\left(\mathrm{k}_{\mathrm{H}}\right)$ acima de 0,55 (Tabela 1), indicam que cadeias agregadas (processo irreversível), ou associadas (processo reversível) estavam presentes ${ }^{[10]}$. A viscosidade da solução foi independente da velocidade de cisalhamento, e um comportamento similar ao Newtoniano é verificado, mas o cisalhamento não foi suficiente para desfazer a agregação de cadeias de celulose em solução ${ }^{[12]}$.

Sendo expressa em unidade de volume por unidade da massa, a [ $\eta]$ está diretamente relacionada ao volume hidrodinâmico da cadeia. Portanto, $[\eta]$ depende da massa molar média e das interações entre os segmentos da cadeia e as moléculas de solvente. Vários parâmetros devem ser levados em conta para avaliar as propriedades de derivados de celulose em solução, como o tipo de substituinte introduzido, o grau de substituição, assim como a distribuição dos grupos substituintes nas posições C2, C3 e C6 da celulose (Figura 1a) ${ }^{[13]}$. A introdução de grupos acetatos na cadeia de celulose pode levar a dois efeitos opostos:

1. Expansão das cadeias ou de estruturas associadas, devido à repulsão estérica entre os grupos acetatos, o que, em princípio aumentaria as interações polímero/solvente ( $\mathrm{LiCl} / \mathrm{DMAc})$;

2. Diminuição das interações polímero/solvente, uma vez que a substituição de $\mathrm{H}$ pelos grupos acetatos diminuiria as interações cel-OH--- $\mathrm{Cl}^{-}$, as quais são importantes no processo de solubilização da celulose em LiCl/DMAc, e provavelmente no de seus derivados ${ }^{[6-8,11]}$.

Para os acetatos com diferentes GS, alguns valores da constante de Huggins, indicaram que cadeias agregadas, ou associadas estavam presentes. Consequentemente, um número reduzido de soluções foi considerado no presente trabalho. A seguir, o comportamento dos acetatos com diferentes GS é discutido.

GS 0,8: Considerando que o GS pode variar até o máximo de 3,0 , para este grau de substituição médio $(<1,0)$, predominam os grupos $\mathrm{OH}$ não substituídos. Os valores da constante de Huggins $\left(\mathrm{k}_{\mathrm{H}}\right.$, Tabela 1) indicam que a $25^{\circ} \mathrm{C}$ pode-se considerar que se tem uma solução molecularmente dispersa. Conforme já constatado ${ }^{[7]}, \mathrm{o}$ carbono mais substituído é o C6 (álcool primário, Figura 1a). Para este GS, praticamente só este grupo estaria substituído. A maior projeção para o meio deste grupo acetato $\left(-\mathrm{C} \mathrm{OCOCH} \mathrm{COCH}_{3}\right)$ pode facilitar interações intramoleculares, as quais podem levar a certo enovelamento da cadeia, e a menor [ $\eta]$. A viscosidade intrínseca obtida para $25{ }^{\circ} \mathrm{C}$ refletiria aproximadamente esta conformação mais enovelada, da cadeia individual. $\mathrm{O}$ valor obtido a $50{ }^{\circ} \mathrm{C}$ não pode, rigorosamente, ser considerado como viscosidade intrínseca, já que se refere a cadeias agregadas/associadas. No entanto, a tendência frente à diminuição $\left(41,0 \mathrm{~mL} \cdot \mathrm{g}^{-1}\right)$, quando comparada ao valor para $25{ }^{\circ} \mathrm{C}\left(45,0 \mathrm{~mL} \cdot \mathrm{g}^{-1}\right)$, pode ser tomada como uma indicação que a estrutura mais compacta das cadeias agregadas/ associadas $^{[14]}$,comparativamente ao novelo aleatório das cadeias individuais ${ }^{[15]}$, pode fazer com que cadeias agregadas ocupem menor volume em solução. Comparativamente, as cadeias de celulose $\left(50{ }^{\circ} \mathrm{C}\right)$ parecem mais agregadas/associadas, e o valor de $[\eta]$ aumenta, relativamente a este acetato.

GS 1,5: Para este grau de substituição médio, o número de hidroxilas substituídas e não substituídas é aproximadamente igual. Em termos de coeficiente, para este acetato, o valor de $\mathrm{k}_{\mathrm{H}}$ obtido (Tabela 1) também indica que a $25{ }^{\circ} \mathrm{C}$ se tem uma solução "verdadeira" com cadeias molecularmente dispersas, enquanto que a $50{ }^{\circ} \mathrm{C}$ se tem cadeias agregadas/associadas. Para este acetato, a diminuição do volume ocupado em solução, devido à formação de agregados/associados, é mais acentuada que para o acetato com GS igual a 0,8 , pois a "viscosidade intrínseca" diminui de 103,0 para 81,0 mL.g ${ }^{-1}$ (Tabela 1), reforçando o que foi discutido anteriormente. Comparando-se os valores de viscosidade intrínseca obtidos a $25{ }^{\circ} \mathrm{C}$, temperatura em que cadeias estão molecularmente dispersas, se observa que a introdução de maior número de grupos acetatos (GS 1,5) leva a uma conformação mais expandida, comparativamente a GS igual a 0,8 , conforme indicado pelo aumento expressivo de 41,0 para 103,0 $\mathrm{mL} \mathrm{g}^{-1}$. Pode-se considerar também que para GS 1,5, C2 e C3 (Figura 1a) começam a ser substituídos, além de C6 (carbono preferencialmente substituído) o que também influencia no valor de $[\eta]$.

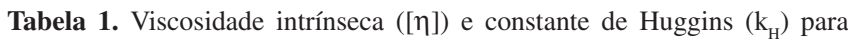
linter mercerizado e acetatos de celulose com GS 0,8; 1,5 e 2,0.

\begin{tabular}{cccc}
\hline Amostra & $\begin{array}{c}\text { Temperatura } \\
\left({ }^{\circ} \mathbf{C}\right)\end{array}$ & $\begin{array}{c}{[\eta]} \\
\left(\mathbf{m L . g}^{-1}\right)\end{array}$ & $\mathbf{k}_{\mathbf{H}}$ \\
\hline \multirow{2}{*}{ Linter mercerizado } & 25 & 98,0 & 1,8 \\
& 50 & 61,0 & 3,8 \\
\cline { 2 - 4 } GS 0,8 & 25 & 45,0 & 0,3 \\
& 50 & 41,0 & 0,8 \\
\cline { 2 - 4 } GS 1,5 & 25 & 103,0 & 0,2 \\
& 50 & 81,0 & 0,6 \\
\cline { 2 - 4 } GS 2,0 & 25 & 101,0 & 0,8 \\
& 50 & 98,0 & 0,7 \\
\hline
\end{tabular}

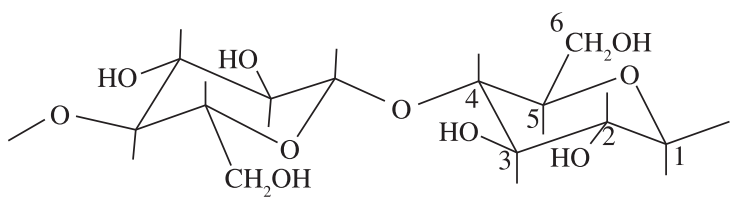

(a)
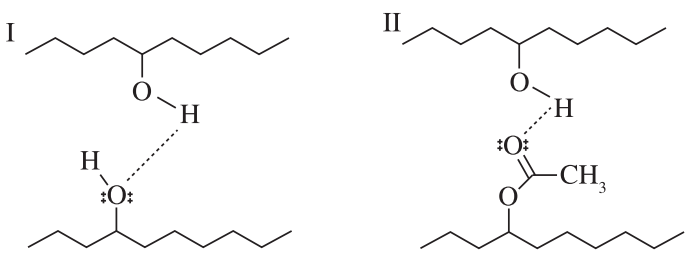

(b)

Figura 1. a) Representação esquemática da celobiose, unidade repetitiva da celulose; e b) representação esquemática da interação entre grupos hidroxilas (I) e a grupo acetato e o grupo hidroxila (II), de cadeias adjacentes. 
GS 2,0: Para este acetato, o número de hidroxilas não substituídas é menor que o de substituídas por grupo acetato. Os valores de $\mathrm{k}_{\mathrm{H}}$ obtidos (Tabela 1) indicam que a $25^{\circ} \mathrm{C}$ as cadeias já se encontram agregadas/associadas. Este resultado indica que a introdução de um maior número de grupos acetatos favorece a agregação/associação. $\mathrm{O}$ maior volume do grupo acetato $\left(-\mathrm{COCH}_{3}\right)$, comparativamente a hidroxila $(-\mathrm{OH})$, pode expandir as cadeias devido à repulsão estérica, além de permitir que a interação entre as cadeias não requisite muita aproximação (Figura 1b, II), comparativamente a interação via ligação hidrogênio entre hidroxilas de cadeias adjacentes (Figura 1b, I), o que pode facilitar a agregação/associação (Figura 1b).

Outra possibilidade é que o menor número de hidroxilas livres prejudica a interação com o sistema de solvente $\mathrm{LiCl} / \mathrm{DMAc}$, favorecendo a interação entre cadeias. Comparando os valores de "viscosidade intrínseca" obtidos para o acetato com GS igual a 2,0 $\left(98,0 \mathrm{~mL} \cdot \mathrm{g}^{-1}\right)$, que na realidade reflete o volume ocupado por cadeias agregadas/associadas, com aquele obtido para GS igual a $1,5\left(81,0 \mathrm{~mL} \cdot \mathrm{g}^{-1}, 50{ }^{\circ} \mathrm{C}\right)$ e $0,8\left(41,0 \mathrm{~mL} \cdot \mathrm{g}^{-1}, 50{ }^{\circ} \mathrm{C}\right)$, que também reflete este tipo de estrutura, se observa maior valor para o primeiro acetato (Tabela 1), indicando que o maior volume dos grupos acetatos leva a maior afastamento entre cadeias que estão interagindo ${ }^{[14]} \mathrm{e}$, portanto, o volume da respectiva estrutura.

Até onde seja do conhecimento dos autores deste estudo, esta abordagem feita é inédita.

\section{Conclusões}

O entendimento das propriedades de soluções de celulose e acetatos de celulose no sistema de $\mathrm{LiCl} / \mathrm{DMAc}$ é essencial para análises feitas a partir de soluções, como espalhamento estático e dinâmico de luz, cromatografia de exclusão por tamanho, assim como para preparação de filmes a partir destas soluções. Através de medidas viscosimétricas, pode-se concluir que acetatos de celulose com diferentes GS requisitam diferentes intervalos de concentrações para se inserir no domínio que se acredita corresponda ao regime diluído, o que também depende da temperatura considerada. Os resultados obtidos permitirão uma escolha melhor embasada do intervalo de concentração mais adequado para preparação de filmes a partir de soluções de acetatos e celulose em $\mathrm{LiCl} / \mathrm{DMAc}$, que corresponde à próxima etapa deste estudo.

\section{Agradecimentos}

A Fundação de Amparo à Pesquisa do Estado de São Paulo (FAPESP) pelo apoio financeiro e pela bolsa (Doutorado Direto) concedida a D. L. M. E. F. agradece ao CNPq pela bolsa de Produtividade em Pesquisa e apoio financeiro.

\section{Referências Bibliográficas}

1. Napolitano, B. A.; Martins, A. F.; Visconte, L. L. Y. \& Nunes, R. C. R. - Polímeros, 14, p.223 (2004).

2. Corti, G. S.; Botaro, V. R.; Gil, L. F. \& Gil, R. P. F. - Polímeros, 14, p.313 (2004).

3. Botaro, V. R.; Santos, C. G. \& Oliveira, V. A. - Polímeros, 19, p.278 (2009).

4. Buchard, W. - Cellulose, 10, p.213 (2003).

5. Roy, C.; Budtova, T. \& Navard, P. - Biomacromolecules, 4, p.259 (2003).

6. Ciacco, G. T.; Morgado, D. L.; Frollini, E.; Possidonio, S. \& El Seoud, O. A. - J. Brazil. Chem. Soc., 21, p.71 (2010).

7. Ass, B. A. P.; Belgacem, M. N. \& Frollini, E. - Carbohydr. Polym., 63, p.19 (2006).

8. Ramos, L. A.; Assaf, J. M.; El Seoud, O. A. \& Frollini, E. Biomacromolecules, 6, p.2638 (2005)

9. Edgar, K. J.; Arnold, K. M.; Blount, W. W.; Lawniczak, J. E. \& Lowman, D. W. - Macromolecules, 28, p.4122 (1995).

10. Huggins, M. L. - J. Am. Chem. Soc. 64, p.2716 (1942).

11. El Seoud, O. A. E.; Marson, G. A.; Ciacco, G. T. \& Frollini, E. Makromol. Chem., 20, p.882 (2000).

12. Kuang, Q.-L.; Zhao, J.-C.; Niu, Y.-H.; Zhang, J. \& Wang, Z.-G. - J. Phys. Chem. B, 112, p.10234 (2008).

13. Clasen, C. \& Kulicke, W.-M. - Prog. Polym. Sci., 26, p.1839 (2001).

14. Morgenstern, B. \& Kammer, H.-W. Polymer, 40, p.1299 (1999).

15. Stevens, M. P. - "Polymer chemistry: an introduction", Oxford University, New York (1990).

Enviado: 11/04/10

Reenviado: $28 / 07 / 10$

Aceito: $24 / 09 / 10$

DOI: 10.1590/S0104-14282011005000019 